Medici, Ch. El fallo "F.A.L" a través de las críticas feministas al derecho. Derecho y Ciencias Sociales. Mayo -Octubre 2021. № 25 .Pgs 45-60. ISSN 1852-2971. Instituto de Cultura Jurídica y Maestría en Sociología Jurídica. Facultad de Ciencias Jurídicas y Sociales. Universidad Nacional de La Plata. Argentina.

\title{
El fallo "F.A.L." a través de las críticas feministas al derecho
}

The ruling "F.A.L" through feminist critiques of the Law

Chantal Medici

\section{Resumen}

En el año 2012 la Corte Suprema de Justicia emitió el fallo “F.A.L.”, que brindó certeza jurídica acerca de la legalidad de la interrupción del embarazo en los casos previstos por la ley, definió los alcances de la protección de la vida intrauterina y su arreglo con los compromisos internacionales y definió las responsabilidades del Estado. Luego de la aprobación de la ley 27.610 de Acceso a la Interrupción Legal del Embarazo, sectores conservadores iniciaron una multitud de acciones, muchas de las cuales esgrimen los mismos argumentos sobre los cuales la Corte Suprema ya sentó posición.

El presente artículo repasa los antecedentes del fallo "F.A.L." y las definiciones de la sentencia y ofrece un análisis de los aportes que puede realizar a las críticas feministas al Derecho.

Palabras clave: Interrupción del Embarazo ; “F.A.L.”; críticas feministas al derecho

\begin{abstract}
In 2012 the Supreme Court of Justice issued the ruling "FAL", which provided legal certainty about the legality of termination in the cases provided by law, defined the standards of the protection of intrauterine life and its arrangement with the international commitments and defined the responsibilities of the state. After the approval of Law 27,610 on Access to Legal Interruption of Pregnancy, conservative sectors initiated a multitude of legal actions, many of which use the same arguments on which the Supreme Court has already established a position. This article reviews the background to the ruling "F.A.L." and the definitions of the sentence and offers an analysis of the contributions that can be made to feminist critiques of the Law.
\end{abstract}

Key words: interruption of pregnancy ; abortion- "F.A.L." ; feminist critiques ; Argentina

\footnotetext{
- Socióloga (UBA), magíster en Public Policy and Development Management (Georgetown University), doctoranda en Ciencias Sociales (UBA). Correo: chantalmedici2@gmail.com
}

Recibido: 15/3/2021 Publicable: 4/10/2021

https://doi.org/10.24215/18522971e091 
Medici, Ch. El fallo "F.A.L" a través de las críticas feministas al derecho. Derecho y Ciencias Sociales. Mayo -Octubre 2021. № 25 .Pgs 45-60. ISSN 1852-2971. Instituto de Cultura Jurídica y Maestría en Sociología Jurídica. Facultad de Ciencias Jurídicas y Sociales. Universidad Nacional de La Plata. Argentina.

\section{El fallo "F.A.L." a través de las críticas feministas al derecho}

Chantal Medici

\section{Introducción}

Luego de la aprobación de la Ley 27.610 de Acceso a la Interrupción Voluntaria del Embarazo el 30 de diciembre de 2020, una multitud de acciones judiciales fueron iniciadas contra la ley por los sectores conservadores. En los primeros meses del año 2021 una gran cantidad de amparos fueron rechazados directamente por razones procesales ${ }^{1}$. Sin embargo, llama la atención la insistencia en argumentos ya debatidos el fallo "F.A.L. s/ medida autosatisfactiva", dictado por la Corte Suprema de Justicia de la Nación en el año 2012, tanto en el debate parlamentario como en las presentaciones.

El fallo, que reafirmó la autorización de la interrupción del embarazo de una niña de 15 años, quien gestaba como consecuencia de un abuso, brindó certeza jurídica acerca de la legalidad de la práctica, fijó la interpretación de los instrumentos internacionales y de la Constitución Nacional que fueron usados como fundamento para lecturas restrictivas durante todo el siglo XX y los inicios del XXI y ofreció un marco para la protección de la vida intrauterina y su arreglo con los compromisos contraídos por el Estado argentino.

Por otra parte, esta sentencia fue antecedida por una construcción jurídica previa en instancias sub y supranacionales que progresivamente transformó la aplicación práctica de la ley y contribuyó a abrir las puertas al cambio normativo. Un recorrido sobre esta historia evidencia la distancia entre la norma y su aplicación efectiva así como también los razonamientos extrajurídicos, pertenencias identitarias y políticas que exceden el ambiente tribunalicio que sostienen los debates relativos a esta ley.

\footnotetext{
${ }^{1}$ Los argumentos ya rebatidos por la Corte Suprema en el fallo "F.A.L." que serán analizados más abajo fueron sustento de la cautelar que suspendió la aplicación de la ley en la provincia de Chaco (Dellamea Hilda Beatriz y otros c/ Gobierno de la Provincia Del Chaco y/o Ministerio de Salud Pública Del Chaco y/o Ministerio de Educación, Cultura, Ciencia y Tecnología del Chaco s/ medida cautelar, 2021), y fueron utilizados como argumento para solicitar la inconstitucionalidad de la ley 27.610 en otros casos que culminaron en un rechazo de la solicitud (PARTIDO CIUDADANOS A GOBERNAR- DISTRITO CORRIENTES C/ GOBIERNO DE LA PROVINCIA DE CORRIENTES Y MINISTERIO DE SALUD PÚBLICA DE CORRIENTES S/ AMPARO, 2021; JUNTA PROMOTORA DEL PARTIDO "NOS" - DISTRITO CHACO c/ ESTADO NACIONAL S/ACCION MERE DECLARATIVA DE INCONSTITUCIONALIDAD", 2021; FIORE VIÑUALES, MARIA CRISTINA Y OTROS c/ MINISTERIO DE SALUD DE LA NACION s/ACCION MERAMENTE DECLARATIVA DE INCONSTITUCIONALIDAD, 2021).
} 
Medici, Ch. El fallo "F.A.L" a través de las críticas feministas al derecho. Derecho y Ciencias Sociales. Mayo -Octubre 2021. № 25 .Pgs 45-60. ISSN 1852-2971. Instituto de Cultura Jurídica y Maestría en Sociología Jurídica. Facultad de Ciencias Jurídicas y Sociales. Universidad Nacional de La Plata. Argentina.

Dada la actualización de la controversia a partir de las acciones presentadas luego de la sanción de la ley IVE, y debido a que se trata de un fallo de altísimo impacto sobre los derechos de las mujeres y personas con capacidad de gestar, resulta pertinente realizar un repaso de las definiciones que hizo el fallo, en particular en lo que respecta a la lectura de los tratados internacionales a los que se otorgó jerarquía constitucional en la reforma de 1994, a la luz de las críticas feministas al derecho.

En el presente trabajo se detallará, en primer lugar, la legislación vigente en materia de aborto en Argentina al momento de la emisión del fallo, la aplicación real de la norma y se resumirán los principales debates jurídicos que tuvieron lugar hasta el fallo. A continuación, se detallarán los principales antecedentes. Luego, se esquematizarán las principales argumentaciones del fallo y la resolución. Finalmente, se analizará la sentencia junto con la reconstrucción de sus antecedentes y la aplicación práctica de la ley a la luz de las críticas feministas al derecho.

\section{La ley, la práctica y los debates jurídicos}

Hasta el 30 de diciembre de 2020, el Código Penal Argentino tipificaba el aborto como un delito excepto si se realizaba "con el fin de evitar un peligro para la vida o la salud de la madre" o si "el embarazo proviene de una violación o de un atentado al pudor cometido sobre una mujer idiota o demente"2.

De todas formas, durante la mayor parte del siglo XX la norma funcionaba de facto como si las excepciones planteadas en el art. 86 no existieran y la penalización fuera total (Motta y Rodríguez, 2000; Chiarotti, 2006). Quienes solicitaban la práctica se enfrentaban a un "círculo de inacción" estatal que volvía el derecho inaccesible de hecho (Motta y Rodríguez, 2000: 15). A pesar de estas circunstancias, a principios del nuevo siglo se estimó que en el país se inducen un promedio de entre 486.000 y 552.000 abortos al año (Mario y Pantelides, 2009). Las complicaciones derivadas del aborto producido en condiciones de riesgo constituyeron hasta

\footnotetext{
${ }^{2}$ Esta es la redacción que tenía el artículo 86 del Código Penal Argentino antes de la sanción de la ley 27.610 que lo modificó. La actual redacción de la norma mantiene las causales previstas en la formulación anterior durante todo el embarazo, además de legalizar la interrupción durante las primeras 14 semanas de gestación.
} 
Medici, Ch. El fallo "F.A.L" a través de las críticas feministas al derecho. Derecho y Ciencias Sociales. Mayo -Octubre 2021. № 25 .Pgs 45-60. ISSN 1852-2971. Instituto de Cultura Jurídica y Maestría en Sociología Jurídica. Facultad de Ciencias Jurídicas y Sociales. Universidad Nacional de La Plata. Argentina.

tiempo reciente la principal causa individual de muerte materna (Ministerio de Salud de la Nación, s/fa). En el año 2016 se registraron 39.025 egresos por aborto en los hospitales públicos (Ministerio de Salud de la Nación, s/fb). Datos presentados por el Ministerio de Salud en el año 2018 estimaron que al incluir el sistema privado de salud esta cifra ascendería a 70 mil (Rubinstein, s/f).

Paralelamente a estos obstáculos burocráticos, la legalidad de estos permisos fue frecuentemente puesta en duda por argumentos basados ya sea en las ambigüedades de su redacción, o en los tratados y convenciones que refieren a la protección de la vida humana. En primer lugar, debido a la imprecisa redacción de la ley, hasta la emisión del fallo "F.A.L." hubo posiciones que argumentaron que la práctica sólo estaba permitida en casos en que la víctima de abuso fuera una mujer con discapacidad ${ }^{3}$.

Respecto de aquellos casos en que hubiera riesgo para la vida o salud de la persona gestante, se debatió si debía haber un cierto grado de riesgo, y si este fuera inminente, o sólo probable. Algunas posiciones señalaron que, de acuerdo con el principio de legalidad, que obliga a tomar la interpretación menos punitiva de la ley, es inadecuado requerir un cierto nivel de riesgo o de probabilidad de daño para el acceso al aborto legal (Ferrante, 2011: 365).

Desde la reforma constitucional de 1994, los fundamentos más importantes de las posiciones en contra de la legalidad del aborto se basaron en los tratados internacionales a los que se les acababa de dar rango constitucional. Se interpretó que algunos de éstos consagraban el derecho a la vida desde la concepción, y que el mismo se jerarquizaba por sobre otros derechos de la persona gestante. Se citó principalmente la Convención Americana sobre Derechos Humanos, cuyo artículo 4.1 establece que "toda persona tiene derecho a que se respete su vida. Este derecho estará protegido por la ley y, en general, a partir del momento de la concepción. Nadie puede ser privado de la vida arbitrariamente". Se referenciaba también la Convención de los Derechos del Niño, que en su artículo 6 establece que "Los Estados parte reconocen que todo niño tiene derecho intrínseco a la vida" (Faerman, 2008).

\footnotetext{
${ }^{3}$ La ambigua formulación se debe a que la ley se basó en una versión francesa de un proyecto suizo, en el que se utilizaban las dos palabras que se usan en alemán para nombrar la violación: el abuso violento (notzucht), y el de una mujer mentalmente discapacitada, enferma o incapaz de consentir (sch:andung). A pesar de que la traducción es correcta, al importarse en una tradición normativa que no distingue ambas figuras, dio lugar a que se pensara que el aborto era solamente legal si la víctima tenía estas condiciones (Soler, 1945: 114). Esta interpretación quedó incluso plasmada en algunas normas, como el primer protocolo de la Ciudad de Buenos Aires, dictado en el año 2007 (Resolución 1174 del 28 de mayo de 2007, Ministerio de Salud de la Ciudad Autónoma de Buenos Aires).
} 
Medici, Ch. El fallo "F.A.L" a través de las críticas feministas al derecho. Derecho y Ciencias Sociales. Mayo -Octubre 2021. № 25 .Pgs 45-60. ISSN 1852-2971. Instituto de Cultura Jurídica y Maestría en Sociología Jurídica. Facultad de Ciencias Jurídicas y Sociales. Universidad Nacional de La Plata. Argentina.

Asimismo, fue frecuentemente referido el artículo 75 inc. 23 de la Constitución Nacional, que encomienda al Congreso "dictar un régimen de seguridad social especial e integral en protección del niño en situación de desamparo, desde el embarazo hasta la finalización del período de enseñanza elemental, y de la madre durante el embarazo y el tiempo de lactancia" (Faerman, 2008).

\section{Antecedentes del fallo "F.A.L."}

Junto con este debate teórico de argumentos acerca de la legalidad del aborto, desde el nuevo milenio diversas sentencias provinciales y supranacionales, así como también Observaciones finales y Recomendaciones de los Comités de las Naciones Unidas conformaron importantes antecedentes del fallo de la Corte.

El más importante de ellos es "L.M.R. c/ Argentina", que fue querellado ante el Comité de Derechos Humanos de Naciones Unidas. Se trató de una joven discapacitada que procuró la interrupción legal de su embarazo, que era producto de una violación, y que fue acompañada por organizaciones feministas a través de múltiples obstáculos burocráticos interpuestos por agentes públicos. En el dictamen del año 2011 el Comité consideró que se violaron los artículos 3 y 17 del Pacto Internacional de Derechos Civiles y Políticos, es decir, el derecho a la igualdad y el derecho a no ser objeto de injerencias arbitrarias en la vida privada. También consideró que se violó el artículo 7, el derecho a no ser sometido a tratos crueles, inhumanos o degradantes al serle negada la interrupción de un embarazo producto de un abuso.

En esos años se emitieron también las primeras sentencias firmes a nivel provincial que autorizaron a jóvenes discapacitadas a interrumpir embarazos producto de una violación. Algunos de estos importantes casos culminaron en la Supremas Cortes de la Provincia de Buenos Aires (R. , L.M. , 'NN Persona por nacer. Protección. Denuncia, 2006), ${ }^{4}$ Mendoza (C.S.M. y otros, 06) y Entre Ríos (Defensora de PY.M. $\mathrm{N}^{\circ}$ (en repr. de persona por nacer) s/medida cautelar de protección de persona, 07). Algunos fallos similares fueron sancionados también por instancias inferiores (S.G.N. s/ situación, 2008) ${ }^{5}$.

\footnotetext{
${ }^{4}$ Se trata del célebre caso "L.M.R.”, que luego será litigado en el Comité de Derechos Humanos de la ONU, que culminó con una condena al Estado argentino, como se describirá más adelante. Los médicos se negaron a realizar el aborto, que fue llevado adelante en la clandestinidad.

${ }^{5}$ Los dos párrafos sobre la construcción subnacional siguen los argumentos del artículo escrito por Ramos et al. (2009).
} 
Medici, Ch. El fallo "F.A.L" a través de las críticas feministas al derecho. Derecho y Ciencias Sociales. Mayo -Octubre 2021. № 25 .Pgs 45-60. ISSN 1852-2971. Instituto de Cultura Jurídica y Maestría en Sociología Jurídica. Facultad de Ciencias Jurídicas y Sociales. Universidad Nacional de La Plata. Argentina.

Otra serie de decisiones se pronunciaron acerca de abortos practicados sobre niñas o mujeres víctimas de abuso sexual que no tenían discapacidad. Constituyeron importantes precedentes, ya que antes del dictamen "F.A.L." una parte importante del debate jurídico giraba todavía en torno a la legalidad de la práctica en esas circunstancias. Una de estas sentencias se emitió en Mar del Plata (O., M.V. s/ Víctima de abuso sexual, 2007) y otra de Río Negro (Asesora de Menores Penal s/ pedido, 2008).

Asimismo, hubo algunos casos que refirieron al inc. 1 del art. 86, es decir, aquello en los que el embarazo representaba un riesgo para la salud (C.P.d. P.,A.K. s/ autorización, 2005). En esta etapa comenzaron a discutirse solamente situaciones en que la gestación afectaba la integridad física ${ }^{6}$.

Otro tipo de precedente importante fueron las Observaciones Finales y Recomendaciones de los Comités de las Naciones Unidas ${ }^{7}$. Los organismos expresaron preocupación por las altas tasas de embarazo adolescente, de muerte por aborto, exhortaron a garantizar el acceso efectivo en los casos en que es legal y llamaron la atención sobre los largos procedimientos para la práctica, especialmente en el caso de violación, indicaron que se tomaran medidas para reducir los casos fatales y exigieron la implementación uniforme de estas en todo el territorio (Comité de Derechos Humanos, 2000, párr. 14; 2010, párr. 13; 2016, párrs. 11 y 12; Comité de los Derechos del Niño, 2010, párrs. 58 y 59, incs. d y e; Comité para la Eliminación de la Discriminación contra la Mujer, 2010, párrs. 37 y 38; 2016, párr. 33 inc. c; Comité de Derechos Económicos, Sociales y Culturales, 2011, párr. 22).

Como se verá a continuación, algunos de estos documentos, junto con el fallo "L.M.R. c/ Argentina" fueron citados por la Corte Suprema en "F.A.L." para sostener sus argumentos (Comité de Derechos Humanos, 2010, párr. 13; Comité de los Derechos del Niño 2010, párr. 58 y 59. d, párr. 12 y 13 del fallo $)^{8}$.

\footnotetext{
${ }^{6}$ Fue famoso también el caso "A.M.A", que trató de una mujer a quien se le diagnosticó cáncer mientas descubrió que estaba embarazada. Murió junto a su hijo, pocas semanas luego de dar a luz, tras serle denegados tanto el aborto legal como la quimioterapia que era incompatible con la gestación. Luego de su deceso tuvo lugar la acción llamada "Requerimiento de Instrucción Fiscal N 1 referido a la muerte de A.M.A.".

${ }^{7}$ Estos organismos son las voces más autorizadas sobre la interpretación de los tratados internacionales, y se celebran sesiones periódicas sobre la aplicación de estos en cada uno de los Estados miembros, a los que acuden representantes del Estado y de la Sociedad Civil, presentando respectivos informes. El resultado son documentos que guían al Estado acerca de cómo accionar sobre determinados temas (ONU, s/f).

${ }^{8}$ Emitido con posterioridad a la sentencia "F.A.L." también es de suma importancia para estos debates el fallo "Caso Artavia Murillo y Otros ('Fecundación in Vitro') vs. Costa Rica" del año 2012, emitido por la Corte Interamericana, que trató la prohibición costarricense de la técnica de fertilización in vitro y realizó una serie de aclaraciones sobre la protección de la vida intrauterina en el ámbito interamericano y su armonización con otros derechos. La sentencia reafirmó que la frase "en general" incluida en el art. 4.1 de la Convención Interamericana sobre Derechos Humanos, que había sido utilizado como sustento de la prohibición ${ }^{8}$, en realidad implica la previsión de posibles excepciones (párr. 188). También indicó que existe "un reconocimiento internacional y
} 
Medici, Ch. El fallo "F.A.L" a través de las críticas feministas al derecho. Derecho y Ciencias Sociales. Mayo -Octubre 2021. № 25 .Pgs 45-60. ISSN 1852-2971. Instituto de Cultura Jurídica y Maestría en Sociología Jurídica. Facultad de Ciencias Jurídicas y Sociales. Universidad Nacional de La Plata. Argentina.

\section{El fallo "F.A.L."}

En el año 2012 la Corte Suprema emitió el fallo "F.A.L. s/ medida autosatisfactiva", en que reafirmó la autorización de la interrupción del embarazo de una niña de 15 años que gestaba como consecuencia de un abuso. La sentencia brindó certeza jurídica acerca de la legalidad de la práctica, fijó la interpretación de los instrumentos internacionales y de la Constitución Nacional que habían sido usados como fundamento para lecturas restrictivas y ofreció un marco para entender la protección de la vida intrauterina y su arreglo con los compromisos contraídos por el Estado argentino.

A.F., en representación de su hija A.G. de 15 años, solicitó a la justicia penal de la provincia de Chubut, en la que se tramitaba la causa contra O.C por la violación de A.G. que se dispusiera la interrupción del embarazo de la niña.

El 3 de diciembre había efectuado la denuncia por violación ante el ministerio público de la provincia de Chubut, entonces A.G cursaba la octava semana de gestación. El juez penal se declaró incompetente y A.F. inició una medida autosatisfactiva que fue rechazada en la primera instancia y en la cámara. El superior tribunal revocó la decisión el 8 de marzo de 2010, con basamento en el art. 86 del Código Penal. La práctica fue llevada adelante el 11 de marzo de 2010.

La decisión fue de todas formas recurrida por el Asesor general subrogante de la provincia de Chubut, que fue otorgado. Fundó sus argumentos en que la niña no era "idiota o demente" (SIC), interpretó que del art. 75 de la Constitución Nacional se desprende la protección de la vida desde la concepción, citó los artículos 1, 3 y 4 de la Declaración Americana de los Deberes y Derechos del Hombre (DADDH), los arts. 3 y 6 de la Declaración Universal de los Derechos Humanos, el art. 6 del Pacto Internacional de los Derechos Civiles y Políticos; Preámbulo, art .1 y art. 6 de Convención sobre los Derechos del Niño, los cuales refieren al derecho a la vida y el derecho al reconocimiento de la personalidad jurídica de la persona.

\footnotetext{
comparado del concepto de protección gradual e incremental de la vida en la etapa prenatal" (párr. 256) y subrayó que no existe una concepción consensuada sobre el comienzo de la vida humana, y que por lo tanto no se puede otorgar preminencia a un cierto tipo de literatura científica sobre otra, ya que esto implicaría imponer un timo de creencias específicas a otras personas que no las comparten (párr. 185). Recordó asimismo que el Comité para la Eliminación de la Discriminación contra la Mujer aclaró que los principios de igualdad y no discriminación exigen privilegiar los derechos de la mujer embarazada sobe el interés de proteger la vida en formación (párr. 277).
} 
Medici, Ch. El fallo "F.A.L" a través de las críticas feministas al derecho. Derecho y Ciencias Sociales. Mayo -Octubre 2021. № 25 .Pgs 45-60. ISSN 1852-2971. Instituto de Cultura Jurídica y Maestría en Sociología Jurídica. Facultad de Ciencias Jurídicas y Sociales. Universidad Nacional de La Plata. Argentina.

El fallo de la Corte, en primer lugar, indicó que es irrazonable la interpretación según la cual serían legales solamente los embarazos producto de una violación sobre mujeres discapacitadas y no sobre cualquier mujer (párr. 15 del fallo). Puntualizó también que obligar a las víctimas a llevar a término un embarazo causado por una violación a sus derechos más fundamentales sería contrario al principio de dignidad de las personas (párr. 16) ${ }^{9}$.

Fijó también la interpretación de tratados y convenciones y de la Constitución Nacional que ocasionaron debates y ofreció un marco para entender la protección de la vida intrauterina y su arreglo con los compromisos internacionales contraídos por el Estado argentino. Indicó que el art. 75 inc. 23 de la Constitución se creó con miras de dictar un marco normativo relativo a la seguridad social y no uno punitivo, y por lo tanto no puede ser sustento de la penalización, como plantearon hasta entonces algunas posiciones (párr. 9).

Señaló que no se deduce, ni del art. 1 de la Declaración Americana de los Derechos y Deberes del Hombre ni del art. 4 de la Convención Americana sobre Derechos humanos, la prohibición total del aborto en todo tipo de circunstancias, dado que estas normas fueron formuladas especialmente de tal forma que no se derivara de ellas la invalidez de este (párr. 10 del fallo). Sostuvo esta postura en la cita del caso "Baby Boy" de la CIDH ("Baby boy", 1981). El art. 3 de la Convención Americana no puede interpretarse de forma aislada al art. 4.

Respecto de los art. 3 y 6 de la Declaración Universal de los Derechos Humanos, entendió que no puede deducirse de ellos una visión restrictiva del art. 86, ya que deben comprenderse a la luz del art. 1 (derecho a la igualdad) (párr. 11).

Con relación al art. 6 del Pacto Internacional de Derechos Civiles y Políticos, indicó que el Comité de Derechos Humanos de las Naciones Unidas manifestó en repetidas ocasiones su postura relativa a que debe permitirse el aborto en caso de violación. Respecto a Argentina, expresó preocupación por la interpretación restrictiva del art. 86 del Código Penal (Comité de los Derechos Humanos, 2000b, 2000c; 2004, 2010) (párr. 12).

En cuanto a la Convención de los Derechos del Niño, señaló que el Preámbulo también fue redactado de tal forma que pudieran adoptarlo los países que admiten el aborto en algunas circunstancias. De hecho, el Comité de los Derechos del Niño ha requerido a los Estados que no contemplan la legalidad del aborto en caso de violación que reformen su normativa (Comité de Derechos del Niño, 1999, 2001, 2010) (párr. 13).

\footnotetext{
${ }^{9}$ Estos derechos están consagrados en el artículo 11 de la Convención Americana sobre Derechos Humanos, en el artículo $1^{\circ}$ de la Declaración Universal de los Derechos Humanos y en los preámbulos del Pacto Internacional de Derechos Civiles y Políticos y de la Declaración Americana de los Derechos y Deberes del Hombre.
} 
Medici, Ch. El fallo "F.A.L" a través de las críticas feministas al derecho. Derecho y Ciencias Sociales. Mayo -Octubre 2021. № 25 .Pgs 45-60. ISSN 1852-2971. Instituto de Cultura Jurídica y Maestría en Sociología Jurídica. Facultad de Ciencias Jurídicas y Sociales. Universidad Nacional de La Plata. Argentina.

El art. 2 de la ley 23.849, que estipula que el artículo 1 de la Convención "debe interpretarse en el sentido que se entiende por niño todo ser humano desde el momento de la concepción" no es una reserva en términos del art. 2 de la Convención de Viena sobre el Derecho de los Tratados que altere el alcance en que rige el tratado en la normativa interna a través del art. 75 inc. 22 de la Constitución Nacional. Argentina formuló una reserva respecto del art. 22, pero sólo una declaración interpretativa respecto del art. 1 (Comisión de Derecho Internacional, 1999)

Definió también que el artículo 86 se armoniza con el Sistema Interamericano de Derechos Humanos de acuerdo con lo consagrado en sus Tratados y Convenciones (párr. 117) ${ }^{10}$. Asimismo, indicó que la solicitud de requisitos no previstos por la ley por parte del personal estatal sería una práctica contra legem, y solicitó al Poder Ejecutivo que dicte protocolos para el acceso al derecho.

\section{"F.A.L" y las críticas feministas al Derecho}

Desde una variedad de perspectivas diferentes, los feminismos llamaron la atención sobre los sesgos androcéntricos del Derecho y elaboraron propuestas acerca de cómo superarlo. Un análisis del fallo junto con sus antecedentes y el contexto en que fue emitido aporta a diferentes postulados y aspectos que fueron enfocados por estas críticas feministas al Derecho.

Olsen (2009) separó estas propuestas en tres vertientes. Dado que el Derecho se presenta a sí mismo como racional, objetivo, abstracto y universal, la primera de ellas, el reformismo legal, denuncia que en verdad la disciplina no cumple estas cualidades y realiza aportes para que alcance este ideal. Las segundas concuerdan con la idea de que el derecho sea racional, objetivo

\footnotetext{
${ }^{10}$ El fallo hace referencia a los siguientes derechos: el derecho a la vida (art. 1 de la Declaración Americana de los Derechos y Deberes del Hombre [en adelante, DADDH], 1948; art. 4.1 de la Convención Americana de los Derechos Humanos [en adelante, CADH], 1969; art. 3.a Convención Interamericana para Prevenir, Sancionar y Erradicar la Violencia contra la Mujer "Convención de Belém do Pará" [en adelante, Convención de Belém do Pará], 1994; a la salud (art. 11, DADDH, 1948, y art. 10 Protocolo Adicional a la Convención Americana en materia de Derechos Económicos, Sociales y Culturales, "Protocolo de San Salvador", 1948), a la libertad y seguridad personales (art. 1, DADDH, 1948; art. 7.1, CADH, 1969; art. 3.c, Convención de Belém do Pará, 1994), a la integridad física, psíquica y moral (art. 5.1, CADH, 1969; art. 3.b, Convención de Belém do Pará, 1994), a no ser sometido a torturas ni a penas o tratos crueles, inhumanos o degradantes (art. 5.2., CADH, 1969; art. 3.d., Convención de Belém do Pará, 1994), a la igualdad y no discriminación (art. 2, DADDH, 1948; art. 1.1 y 24 , CADH, 1969; art. 3.f., Convención de Belém do Pará, 1994), a la dignidad (art. 17, DADDH, 1948; art. 11.1, CADH, 1969; y art. 3.e., Convención de Belém do Pará, 1994), a no ser objeto de injerencias arbitrarias o abusivas en su vida privada (art. 5, DADDH, 1948; y art. 11.2 y 11.3, CADH, 1969), y a la protección especial a la niñez (art. 7, DADDH, 1948; y art. 19, CADH, 1969). En especial, la Convención de Belém do Pará dispone que la violencia contra las mujeres comprende la violencia sexual (art. 2), consagra el derecho de las mujeres a una vida libre de violencia (art. 3), y reconoce que la violencia contra las mujeres impide y anula el ejercicio libre pleno de sus derechos civiles, políticos, económicos, sociales y culturales (art. 5) (1994).
} 
Medici, Ch. El fallo "F.A.L" a través de las críticas feministas al derecho. Derecho y Ciencias Sociales. Mayo -Octubre 2021. № 25 .Pgs 45-60. ISSN 1852-2971. Instituto de Cultura Jurídica y Maestría en Sociología Jurídica. Facultad de Ciencias Jurídicas y Sociales. Universidad Nacional de La Plata. Argentina.

y universal, y por lo tanto masculino y patriarcal. Proponen por lo tanto vincular la incorporación de las demandas de las mujeres en el derecho a cambios más radicales.

Para la tercera vertiente, la "teoría crítica jurídica feminista", el derecho es tan racional, objetivo, abstracto y universal como irracional, subjetivo, concreto y particular. La afirmación de que el derecho es universal se basa en la creencia de que de unos pocos principios generales se deducen los fundamentos para resolver casos particulares, en realidad el derecho está formado por una acumulación de una gran cantidad de normas específicas y algunos principios muy generales. El derecho tampoco es objetivo: en todas las decisiones legales hay posiciones políticas.

En línea con este planteo, Abramovich (1991) ya había señalado que las sentencias son el resultado de un proceso de lucha de argumentos y posturas tanto jurídicas como extrajurídicos, que circulan tanto al interior como al exterior del tribunal. La reconstrucción de los antecedentes del fallo y los debates políticos durante el siglo XX evidencian cómo los posicionamientos jurídicos están permeados de posturas religiosas e ideológicas. La insistencia de los sectores conservadores en los posicionamientos ya rebatidos por la Corte Suprema, a través de una multitud de acciones judiciales, refuerza este postulado. Adicionalmente, se hace visible la distancia entre la imagen que el derecho y la práctica jurídica tienen de sí mismos, entre lo escrito en la ley y la aplicación efectiva que fuera señalada repetidamente por las críticas feministas al derecho (Olsen, 2009; Fascio, 1999).

Bartlett (1990), por su parte, postuló que deben identificarse los puntos de la doctrina legal que pone en desventaja a las mujeres (la "pregunta por la mujer") y razonar desde un ideal en el que las resoluciones legales son respuestas a dilemas concretos. No opone el método feminista al razonamiento abstracto y deductivo, sino que propone la incorporación de ciertas particularidades contextuales y la recuperación del punto de vista de la mujer. La intervención de la Corte en una materia que se había vuelto abstracta puede constituir una incorporación de la "pregunta por la mujer" y los "problemas de las mujeres" a la práctica judicial, al dictar los lineamientos en que debe aplicarse el art. 86.

Los sesgos androcéntricos de los "Derechos Universales" también fueron abordados por los feminismos. Eisenstein (1988) propuso la búsqueda de estos sesgos dado que, si bien las declaraciones incluyeron a las mujeres, lo hicieron desde una perspectiva androcéntrica. Asimismo, el aborto está atravesado tanto por cuestiones de igualdad formal como de igualdad sustantiva. Por un lado, se trata de una ley penal que afectaba solamente a un grupo de la población (el art. 88 indica que "será reprimida con prisión de uno a cuatro años la mujer que 
Medici, Ch. El fallo "F.A.L" a través de las críticas feministas al derecho. Derecho y Ciencias Sociales. Mayo -Octubre 2021. № 25 .Pgs 45-60. ISSN 1852-2971. Instituto de Cultura Jurídica y Maestría en Sociología Jurídica. Facultad de Ciencias Jurídicas y Sociales. Universidad Nacional de La Plata. Argentina.

causare su propio aborto o consintiere que otro se lo causare"). Por otra parte, al tratarse la adolescente A.G. de un sujeto perteneciente a una doble vulnerabilidad de acuerdo con las "Cien reglas de Brasilia", por su edad y por ser mujer, abarca también cuestiones de igualdad sustantiva.

Por otra parte, reconstruye la relación entre lo público y lo privado, otro tópico central enfocado por los feminismos. De forma muy diversa a otros fallos que basaron sus argumentos en el concepto de privacidad (privacy) como Roe vs. Wade (Poovey, 1992), "F.A.L." se apoya escasamente en encuadres sustantivos (igualdad, dignidad, autonomía) y más fuertemente en encuadres transversales (principio de legalidad, obligaciones estatales respecto de los derechos, etc.) (Pou Giménez, 2019). El acceso a la interrupción legal del embarazo no es una cuestión privada de las personas gestantes sino una responsabilidad del Estado.

\section{Conclusiones}

Pocos fallos constituyen un hito fundamental y un parteaguas en el avance hacia la conquista de un derecho como lo fue el fallo "F.A.L.". Un recorrido sobre sus antecedentes, los debates jurídicos que lo atravesaron, la construcción jurídica subnacional y su inscripción en los tratados internacionales, de acuerdo con los documentos emitidos por los órganos de los tratados desde las críticas feministas al derecho actualiza estas miradas.

Hasta el 2020, aún permaneciendo invariable la norma, existió un desplazamiento importante en la aplicación práctica. Mientras que años atrás la ley funcionaba de facto como si la prohibición del aborto fuera total, y lecturas que postulaban la incompatibilidad de cualquier forma de legalización del aborto con el sistema jurídico argentino tenían amplio consenso, progresivamente a través de los debates y fallos se construyó una lectura que, por el contrario, enmarcó la interrupción del embarazo en la normativa interna y los compromisos internacionales contraídos. Antes de la sanción de la nueva ley, los informes oficiales daban cuenta de una importante ampliación del acceso a la Interrupción Legal del Embarazo (Ministerio de Salud de la Provincia de Buenos Aires, 2020; Coordinación de Salud Sexual, SIDA e ITS, 2019)

Tal como plantearon Olsen (2009) y Abramovich (1991), la distancia entre la ley y la práctica, la evolución de la jurisprudencia y de la implementación efectiva no pueden entenderse sólo a partir de razonamientos jurídicos sino sobre todo a partir de posturas extrajurídicas, religiosas 
Medici, Ch. El fallo "F.A.L" a través de las críticas feministas al derecho. Derecho y Ciencias Sociales. Mayo -Octubre 2021. № 25 .Pgs 45-60. ISSN 1852-2971. Instituto de Cultura Jurídica y Maestría en Sociología Jurídica. Facultad de Ciencias Jurídicas y Sociales. Universidad Nacional de La Plata. Argentina.

y políticas que exceden el ámbito tribunalicio porque son compartidas por amplios sectores de la sociedad.

Tal como Eisenstein (1988) propuso la búsqueda de sesgos androcénticos en los "Derechos Universales", existe una construcción que progresivamente incorpora la "pregunta por la pregunta por la mujer" y "los problemas de las mujeres" en el derecho internacional a través de las Recomendaciones y Observaciones finales de los Comités, los fallos supranacionales, subnacionales y los fallos de la Corte Suprema. Se observa un proceso de construcción de un posicionamiento jurídico más favorable a los derechos de las mujeres a través de los debates, fallos subnacionales e internacionales, Recomendaciones y Observaciones Finales de los Comités de las Naciones Unidas que culmina en el fallo "F.A.L.". A su vez, puede considerarse que esta sentencia, al aclarar los alcances de la protección de la vida intrauterina y el arreglo de las causales con el ordenamiento jurídico internacional, si bien establece definiciones respecto de la normativa anterior, sentó las bases de una lectura de la Constitución Nacional y los tratados que aloje la presente ley ${ }^{11}$.

\section{Referencias}

Abramovich, V. (1991) El complejo de Rock Hudson. Sobre el menosprecio teórico de la práctica judicial y su discurso. En: No Hay Derecho. Año 2. № 4. Buenos Aires.

“Asesora de Menores Penal s/ pedido". Juzgado de Instrucción N 12. General Roca (31 de marzo de 2008)

Artavia Murillo y Otros ("Fecundación in Vitro") vs. Costa Rica. Excepciones preliminares, fondo, reparaciones y costas. Corte Interamericana, 28 de noviembre del año 2012.

“Baby Boy, 2141”. Comisión Interamericana de Derechos Humanos, 6 de marzo de 1981.

Bartlett, K.T. (1990): Feminist Legal Methods. Harvard Law Review, Vol. 103, No. 4, febrero de 1990.

"Cien reglas de Brasilia sobre acceso a la Justicia de las Personas en condición de vulnerabiliad". Brasilia, $4^{\mathrm{a}}$ a 6 de marzo de 2008.Recuperado de https://www.acnur.org/fileadmin/Documentos/BDL/2009/7037.pdf

Chiarotti, S. (2006). El aborto en el marco de los derechos humanos. La situación Argentina. En S. Checa (Comp.). Realidades y coyunturas del aborto. Entre el derecho y la necesidad (pp. 91-110). Buenos Aires, Argentina: Paidós.

\footnotetext{
${ }^{11}$ Con anterioridad al fallo "F.A.L.", en el año 2011 el jurista Marcelo Ferrante afirmaba que existía en el derecho argentino un espacio jurídico para una sentencia del tipo "Roe vs. Wade", es decir, de nuestro marco jurídico no se deducía ni la prohibición ni mucho menos el mandato de una tipificación penal para el aborto (Ferrante, 2011:371)
} 
Medici, Ch. El fallo "F.A.L" a través de las críticas feministas al derecho. Derecho y Ciencias Sociales. Mayo -Octubre 2021. № 25 .Pgs 45-60. ISSN 1852-2971. Instituto de Cultura Jurídica y Maestría en Sociología Jurídica. Facultad de Ciencias Jurídicas y Sociales. Universidad Nacional de La Plata. Argentina.

Código Penal Argentino. Infoleg. Información Legislativa. Recuperado de http://servicios.infoleg.gob.ar/infolegInternet/anexos/15000-19999/16546/texact.htm

Comisión de Derecho Internacional (1999) Anuario de la Comisión de Derecho Internacional. Volúmen II. A/CN.4/SER.A/1999/Add.1.Naciones Unidas. Recuperado el 13/09/2020 de https://legal.un.org/ilc/publications/yearbooks/spanish/ilc_1999_v2_p2.pdf

Comité de Derechos Económicos, Sociales y Culturales. (14 de diciembre de 2011). E /C.12/ARG/CO/3, Examen de los informes presentados por los Estados partes en virtud de los artículos 16 y 17 del Pacto. Comité de Derechos Económicos, Sociales y Culturales. $47^{\circ}$ período de sesiones. Recuperado de http://tbinternet.ohchr.org/_layouts/treatybodyexternal/Download.aspx?symbolno=E/C. 12/ARG/CO/3\&Lang=En

Comité de Derechos Humanos (15 de noviembre de 2000a). Observaciones finales del Comité de Derechos Humanos. Argentina. $70^{\circ}$ Período de Sesiones. CCPR/CO/70/ARG. Nueva York. http://tbinternet.ohchr.org/_layouts/treatybodyexternal/Download.aspx?symbolno=CCP $\underline{\mathrm{R} \% 2 \mathrm{FCO} \% 2 \mathrm{~F} 70 \% 2 \mathrm{FARG} \& \mathrm{Lang}=\mathrm{es}}$

Comité de Derechos Humanos. (12 de agosto de 2004). Observaciones finales del Comité de Derechos Humanos. Gambia. 81 ${ }^{\circ}$ Período de sesiones. CCPR/CO/75/GMB; recuperado de

http://tbinternet.ohchr.org/_layouts/treatybodyexternal/Download.aspx?symbolno=CCP $\underline{\mathrm{R} \% 2 \mathrm{FCO} \% 2 \mathrm{~F} 75 \% 2 \mathrm{FGMB} \& \mathrm{Lang}=\mathrm{en}}$

Comité de Derechos Humanos. (15 de noviembre de 2000c). Observaciones Finales del Comité de Derechos Humanos. Perú. $70^{\circ}$ Período de Sesiones. CCPR/CO/70/PER. Recuperado de

http://tbinternet.ohchr.org/_layouts/treatybodyexternal/Download.aspx?symbolno=CCP R\%2FCO\%2F70\%2FPER\&Lang=es

Comité de Derechos Humanos. (24 de julio de 2000b). Observaciones Finales del Comité de Derechos Humanos. Irlanda. $70^{\circ}$ Período de Sesiones. A/55/40. Recuperado de http://tbinternet.ohchr.org/_layouts/treatybodyexternal/Download.aspx?symbolno=A\%2 F55\%2 F40\%5BVOL.I\%5D(SUPP)\&Lang=en

Comité de Derechos Humanos. (31 de marzo de 2010). Observaciones finales del Comité de Derechos Humanos a Argentina en su $98^{\circ}$ período de sesiones. Nueva York, 31 de marzo de 2010. CCPR/C/ARG/CO/4; Recuperado de http://www2.ohchr.org/english/bodies/hrc/docs/CCPR.C.ARG.CO.4_S.pdf

Comité de los Derechos del Niño (21 de junio de 2010). Examen de los informes presentados por los Estados partes en virtud del artículo 44 de la Convención. CRC/C/ARG/CO/3-4. Comité de los Derechos del Niño. $54^{\circ}$ período de sesiones. Recuperado de http://www2.ohchr.org/english/bodies/crc/docs/co/CRC.C.ARG.CO.3-4_sp.pdf

Comité de los Derechos del Niño. (21 de febrero de 2001). Observaciones Finales del Comité de los Derechos del Niño. Palau. CRC/C/15/Add.149. Recuperado de http://docstore.ohchr.org/SelfServices/FilesHandler.ashx?enc=6QkG1d\%2FPPRiCAqh Kb7yhsgnXZ0ChBsrwmcy8\%2F\%2BFNoDESwV7CzlklT2TCWdv3AoDQIIqX\%2Fip 50s1R20RO2rrXqM3H\%2FwAFZ5WWwstvJPClmogrF0cHc8AGSHXzJCykkewh 
Medici, Ch. El fallo "F.A.L" a través de las críticas feministas al derecho. Derecho y Ciencias Sociales. Mayo -Octubre 2021. № 25 .Pgs 45-60. ISSN 1852-2971. Instituto de Cultura Jurídica y Maestría en Sociología Jurídica. Facultad de Ciencias Jurídicas y Sociales. Universidad Nacional de La Plata. Argentina.

Comité de los Derechos del Niño. Observaciones Finales del Comité de los Derechos del Niño. (24 de agosto de 1999). Chad. CRC/C/15/ Add.107. Recuperado de http://undocs.org/CRC/C/15/Add.107

Comité para la Eliminación de la Discriminación contra la Mujer (16 de agosto de 2010). Observaciones finales del Comité para la Eliminación de la Discriminación contra la Mujer a Argentina. $46^{\circ}$ Período de Sesiones. CEDAW/ARG/CO/6. Nueva York. Recuperado de http://www.acnur.org/t3/fileadmin/Documentos/BDL/2012/8408.pdf?view=1

Convención Interamericana para Prevenir, Sancionar y Erradicar la Violencia contra la Mujer. Recuperado de http://www.oas.org/juridico/spanish/tratados/a-61.html

Coordinación de Salud Sexual, SIDA e ITS (2019): "Salud sexual y Reproductiva en la Ciudad de Buenos Aires. Situación y Respuesta 2019". Ministerio de Salud de la Ciudad de Buenos Aires. Access 21 January 2021, https://www.buenosaires.gob.ar/sites/gcaba/files/situacion_y_respuesta_en_ssyr._caba_ 2019_para_publicar.pdf

“C.P.d. P.,A.K. s/ autorización”. Suprema Corte de la Provincia de Buenos Aires (6 de mayo de 2005

"C.S.M. y otros. Corte Suprema de Justicia de la Provincia de Mendoza”. Sala $1^{\circ}$ (28 de agosto de 2006)

Declaración Americana de los Derechos y Deberes del Hombre. Organización de los Estados Americanos (OEA). Bogotá (1948). Recuperado de http://www.oas.org/es/cidh/mandato/Basicos/declaracion.asp

Declaración Universal de los Derechos Humanos. Organización de las Naciones Unidas. París, Francia. (10 de diciembre de 1948). Recuperado de http://www.un.org/es/universaldeclarationhuman-rights/

Defensora de PY.M. $\mathrm{N}^{\circ}$ (en repr. de persona por nacer) s/medida cautelar de protección de persona. S.T. de Entre Ríos (20 de septiemre de 2007)

"Dellamea Hilda Beatriz y otros c/ Gobierno de la Provincia Del Chaco y/o Ministerio de Salud Pública Del Chaco y/o Ministerio de Educación, Cultura, Ciencia y Tecnología del Chaco s/ medida cautelar" Juzgado Civil y Comercial nro 19 de Resistencia, Chaco, 28 de enero 2021. Recuperado el $09 / 03 / 2021$ de http://www.saij.gob.ar/FA21110000?utm_source=newslettersemanal\&utm_medium=email\&utm_term=semanal\&utm_campaign=jurisprudenciaprovincial

Eisenstein, Zillah R. (1988) The Female Body and The Law. University of California.

Faerman, R. (2008). Algunos debates constitucionales sobre aborto. Revista Lexis Nexis. Recuperado de http://www.juragentium.org/topics/latina/es/faerman.htm

Fascio, A. (1999): Hacia otra teoría crítica del Derecho. En L. Fríes y A. Fascio (comp.): Género y Derecho. Santiago: LOM Ediciones. La Morada

Ferrante, M. (2011). Sobre la permisividad del aborto en el derecho penal argentino. En P. Bergallo (Comp.). Aborto y justicia reproductiva (pp. 363-375). Buenos Aires, Argentina: Editores del Puerto. 
Medici, Ch. El fallo "F.A.L" a través de las críticas feministas al derecho. Derecho y Ciencias Sociales. Mayo -Octubre 2021. № 25 .Pgs 45-60. ISSN 1852-2971. Instituto de Cultura Jurídica y Maestría en Sociología Jurídica. Facultad de Ciencias Jurídicas y Sociales. Universidad Nacional de La Plata. Argentina.

FIORE VIÑUALES, MARIA CRISTINA Y OTROS c/ MINISTERIO DE SALUD DE LA NACION S/ACCION MERAMENTE DECLARATIVA DE INCONSTITUCIONALIDAD. Expte 4290/2020. Juzgado Federal de Salta nro 1, 20 de enero de 2021. Recuperado el 09/03/20221 de http://palabrasdelderecho.com.ar/articulo/2191/Rechazaron-una-cautelar-parasuspender-la-Ley-de-Interrupcion-Voluntaria-del-Embarazo-

JUNTA PROMOTORA DEL PARTIDO "NOS" - DISTRITO CHACO c/ ESTADO NACIONAL S/ACCION MERE DECLARATIVA DE INCONSTITUCIONALIDAD”, Expte. $N^{o}$ 76/2021. Juzgado Federal de Resistencia. 05/02/2021. Recuperado el 09/08/2021

https://www.erreius.com/Jurisprudencia/documento/20210217130346623/accion-deinconstitucionalidad-rechazo-in-limine-ley-27-610-interrupcion-voluntaria-delembarazo-partidos-politicos-legitimacion-activa

Mario, S. y Pantelides, E.A. (2009). Estimación del aborto inducido en Argentina. Notas de Población. Año XXXV (87), 95-120. Recuperado de http://repositorio.cepal.org/handle/11362/12842

Ministerio de Salud (s/fa) Análisis de la mortalidad materno - infantil 2007-2016. Recuperado el 04/08/2020 de http://www.msal.gob.ar/images/stories/bes/graficos/0000001229cntanalisis-mmi-2007-2016.pdf

Ministerio de Salud de la Nación (s/fb) Egresos hospitalarios por grupo diagnóstico. Recuperado el 02/09/2020 de http://www.deis.msal.gov.ar/index.php/causas-egresos/

Ministerio de Salud de la Provincia de Buenos Aires (2020) Informe de gestión. Estrategia de implementación de la interrupción legal del embarazo en la Provincia de Buenos Aires. Período enero a junio 2020. Access February 2nd, 2020, http://www.ms.gba.gov.ar/sitios/srpr/files/2020/10/Informe-ILE1016.pdf

Motta, C. y Rodríguez, M. (2000). Mujer y justicia. El caso argentino. Serie de Informes del Fondo de Apoyo Operativo de Género de LAC No. 3.

“O., M.V. s/ Víctima de abuso sexual” (Trib. Men. №1 de Mar del Plata 14 de 02 de 2007).

Olsen, F. (2009) El sexo del derecho. En R. Ávila Santamaría, J. Salgado y L. Valladares (comp.) (2009) El género en el derecho. Ensayos críticos. Quito: Ministerio de Justicia y Derechos Humanos

Organización de las Naciones Unidas (ONU) (s/f) Comité de los Derechos Humanos. Vigilancia del ejercicio de los derechos civiles y políticos, documento electrónico: https://www2.ohchr.org/spanish/bodies/hrc/ , acceso el 21 de octubre de 2018.

Pacto Internacional de Derechos Civiles y Políticos. Asamblea General de las Naciones Unidas (23 de marzo de 1966). Oficina del Alto Comisionado de Naciones Unidas para los Derechos Humanos. Recuperado http://www.ohchr.org/SP/ProfessionalInterest/Pages/CCPR.aspx

"PARTIDO CIUDADANOS A GOBERNAR- DISTRITO CORRIENTES C/ GOBIERNO DE LA PROVINCIA DE CORRIENTES Y MINISTERIO DE SALUD PÚBLICA DE CORRIENTES S/ AMPARO" Expte. EXP 209906/21. Juzgado de Ejecución Tributaria de la Provincia de Corrientes. 5 de febrero de 2021. Recuperado el 09/03/2021 de http://www.juscorrientes.gov.ar/wp-content/uploads/prensa/pdf/2021/RESOLUCIONEJECUCION-TRIBUTARIA.pdf 
Medici, Ch. El fallo "F.A.L" a través de las críticas feministas al derecho. Derecho y Ciencias Sociales. Mayo -Octubre 2021. № 25 .Pgs 45-60. ISSN 1852-2971. Instituto de Cultura Jurídica y Maestría en Sociología Jurídica. Facultad de Ciencias Jurídicas y Sociales. Universidad Nacional de La Plata. Argentina.

Poovey, M. (1992): El tema del aborto y la muerte del hombre. Buenos Aires: CECYM.

Pou Giménez, F. (2019): La sentencia 'F.A.L'. y la despenalización por indicaciones: una encrucijada en el tratamiento jurídico del aborto en América Latina. Recuperado el $11 / 09 / 2020$ https://www.academia.edu/38766243/La_sentencia_F_A_L_y_la_despenalizaci\%C3\% B3n_por_indicaciones_una_encrucijada_en_el_tratamiento_jur\%C3\%ADdico_del_abor to_en_Am\%C3\%A9rica_Latina

Protocolo Adicional a la Convención Americana en materia de Derechos Económicos, Sociales y Culturales, "Protocolo de San Salvador". El Salvador, 17 de noviembre de 1988. Recuperado de http://www.oas.org/es/sadye/inclusion-social/protocolossv/docs/protocolo-san-salvador-es.pdf

Ramos, Silvina, Bergallo, Paola, Romero, Mariana y Arias Feijóo, Jimena (2009). El acceso al aborto permitido por la ley: un tema pendiente de la política de derechos humanos en Argentina, en CELS, Derechos Humanos en Argentina. Informe Anual 2009. Buenos Aires: Siglo XXI, pp. 451-491, https://www.cels.org.ar/web/wpcontent/uploads/2016/06/Debate-2-El-acceso-al-aborto-permitido-por-la-ley-un-temapendiente-de-la-politica-de-DDHH-en-la-Argentina.pdf, acceso el 15 de noviembre de 2019.

Resolución 1174 del 28 Mayo de 2007, Ministerio de Salud de la Ciudad Autónoma de Buenos Aires. Retrieved 08/02/2020 from: https://boletinoficial.buenosaires.gob.ar/normativaba/norma/100573

“R., L.M. , NN Persona por nacer. Protección. Denuncia” (Suprema Corte de Justicia de Buenos Aires 31 de 07 de 2006).

"Requerimiento de Instrucción Fiscal No 1 referido a la muerte de Ana María Acevedo". Recuperado de https://www.womenslinkworldwide.org/en/files/2732/gjo-argentina-amariaacevedo-espdf.pdf

Rubinstein (s/f) "Aportes desde el Ministerio de Salud al debate sobre la ley de IVE en el Honorable Senado de la Nación". Recuperado el 02/09/2020 de https://drive.google.com/file/d/1-j3PzujjIbnTWJ7N-CAQfFr6spMMnFG_/view

"S.G.N. s/ situación.Tribunal de Familia de Bahía Blanca" (1 de octubre de 2008). 